\title{
Impact of Next-Generation Synchrotron Radiation Sources on Materials Research
}

\author{
G. K. Shenoy \\ Advanced Photon Source \\ Argonne National Laboratory \\ Argonne, IL 60439, USA
}

The submitted manuscript has been created by the
University of Chicago as Operator of Argonne
National Laboratory ("Argonne") under Contract
No. W-31-109-ENG-38 with the U.S. Department
of Energy. The U.S. Government retains for itself,
and others acting on its behalf, a paid-up,
nonexclusive, irrevocable worldwide license in
said article to reproduce, prepare derivative works,
distribute copies to the public, and perform
publicly and display publicly, by or on behalf of
the Government.

Submitted to the Proceedings of the Third International Conference on Synchrotron Radiation in Materials Science, January 21-24, 2002, Singapore. 


\begin{abstract}
Three generations of synchrotron radiation sources have revolutionized our understanding of various correlations in the equilibrium phase of materials through x-ray imaging, spectroscopy and scattering techniques. It is anticipated that new sources based on energy-recovery linacs (ERLs) and x-ray freeelectron lasers (FELs) will deliver x-ray pulses that are below a few-hundred femtoseconds in length and have very high coherence. These sources will extend and broaden our current knowledge of materials science. But more importantly, it is expected that these sources will provide the first glimpse of nonequilibrium processes in materials, including nonthermal melting, metal-insulator transitions involving nonequilibrium phases, laser-induced catalytic oxidation, and determining the structure of imperfect nanoparticles lacking crystalline structure.
\end{abstract}

PACS numbers: 42.55.Vc, 41.60.Ap, 78.47.+p, 64.60.My

Key words: Synchrotron radiation, FEL, ERL, femtosecond science, phase transitions. 


\section{Introduction}

The impact of the three generations of synchrotron radiation sources on materials research has been truly profound. The underlying techniques that have been employed are scattering, diffraction, imaging and spectroscopies of materials under different conditions of temperature, magnetic field and high pressure in all three generations of sources. At the third-generation sources, various properties of x-rays are being fully exploited to make revolutionary advances. These include beam brilliance, flux, polarization, coherence, and time structure. In the following, the current status and future advances at the thirdgeneration facilities in some of the key areas are summarized.

The main purpose of most of the investigations is to study the equilibrium behavior of atoms in condensed matter. Different techniques project different aspect of this behavior. Through the use of scattering techniques, one measures the scattering cross section, which is proportional to the dynamical structure factor, $\mathrm{S}(\mathrm{Q}, \omega)$, where $\mathrm{Q}$ and $\omega$ are the transferred momentum and energy during the scattering event. This function is independent of the probe [1]. For example, inelastic x-ray scattering measurements have provided $S(Q, \omega)$ in a large number of systems $[2,3]$. Nuclear resonant inelastic scattering, on the other hand, provides the same information averaged over all Q values [4,5]. Certain techniques in the $(\mathrm{Q}, \mathrm{t})$ space provide a measure of the intermediate scattering factor, $\mathrm{F}(\mathrm{Q}, \mathrm{t})$. These include x-ray diffraction, coherent nuclear resonant scattering, and x-ray photon correlation spectroscopy [6]. The most important measurement in understanding the equilibrium dynamics of any condensed system is the space-time correlation function, $G(r, t)$. This is usually measured with a variety of imaging and microscopic techniques. It should be recognized that $S(Q, \omega)$ is the time Fourier transform of $F(Q, t)$ and is the time and space Fourier transform of $G(r, t)[1]$. This is best visualized through the conjugate phase map shown in Fig. 1. With the use of various techniques at the thirdgeneration synchrotron sources, scientists have made considerable progress in filling most of this map.

Some of the most noteworthy advances shown in Fig. 1 are: 
a. The resolution of real space techniques, such as microscopy, measuring $\mathrm{G}(\mathrm{r}, \mathrm{t})$ has reached submicron levels. The resolution overlaps with those obtained from reciprocal space techniques, such as $\mathrm{X}$-ray diffraction measuring $\mathrm{F}(\mathrm{Q}, \mathrm{t})$.

b. The source brilliance has made it possible to perform time-resolved experiments with subnanosecond resolution using both imaging and diffraction. For example, picosecond diffraction has demonstrated transient structural changes in photoexcited organic solids [7].

c. The source brilliance and time structure of storage-ring fill patterns allow the capability to perform coherent nuclear resonant scattering and nuclear resonant inelastic scattering experiments, which have led to additional dynamical information $[4,5]$.

d. The coherence of undulator radiation has given us the opportunity to perform photon correlation spectroscopy at x-ray wavelengths to probe the dynamics in many hard and soft condensed-matter systems $[6,8]$.

e. X-ray inelastic scattering has provided a new avenue to measure the dynamical structure factor, $\mathrm{S}(\mathrm{Q}, \omega)$, over a broad momentum and energy transfer region partly overlapping with that from neutron and electron inelastic scattering [2,3].

As can be seen from Fig. 1, in spite of the remarkable progress made by third-generation synchrotron sources in the x-ray region, there is limited information in the picosecond-to-femtosecond time domain. Many investigations have recently been carried out in which a system is pumped to an excited state using a femtosecond optical laser pulse followed by probing of the system as a function of time with femtosecond resolution using a variety of tools. Since vibrational periods of nuclei in condensed matter are about $100 \mathrm{fs}$, the system reaches equilibrium dynamics over many picoseconds. With the advent of femtosecond optical laser sources, optical probes are now being successfully applied to study relaxation and correlation behavior of systems in their excited states. The x-ray studies will provide complimentary information, primarily in the understanding of nonequilibrium processes. Unfortunately, no brilliant xray source exists with suitable characteristics to probe electronic and structural properties with 
femtosecond resolution, although progress has been made through the use of plasma excitation sources $[9,10]$. A natural question arises whether a new class of high-brilliance synchrotron radiation sources with femtosecond resolution capability in the x-ray range can be designed.

\section{Development of New Sources}

A natural limitation exists in designing a storage ring with diffraction-limited value for horizontal emittance, and subpicosecond electron bunches [12,13]. A linac, on the other hand, is a nonequilibrium device in which the electron bunch characteristics including its emittance and length can be tailored with appropriate technology. For these reasons, there has been considerable interest in linacs as being future synchrotron sources.

In a linac, the beam emittance is governed by the geometrical size of the electron beam from the cathode gun. Typical numbers for the normalized emittance, $\varepsilon_{\mathrm{n}}$, of modern guns is about $1 \mathrm{~mm} . \mathrm{mrad}$. If the electrons from such a gun are accelerated to $20 \mathrm{GeV}$, the beam eimittance will be reduced by a factor $\gamma$ $\left(\approx 4 \times 10^{4}\right)$ to 25 pm.rad. In addition, pulse lengths of 20-200 fs are technologically feasible. These characteristics of a linac have provided the basis for two types of future sources. One is an energyrecovery linac (ERL) source and the other is an x-ray free-electron laser (FEL). When implemented, these new sources will have a revolutionary impact not only in the field of materials science but also in other areas of science.

The key to the success of an ERL is energy recovery [14]. Since the energy of electrons in a linac is lost at the end of the linac (on a dump), it is important to recover the energy of the electrons and reuse it with minimum energy loss. In the current conceptual designs, properly tailored low-emittance femtosecond bunches of electrons are accelerated to high energy in a superconducting linac before they pass through a set of undulators to produce synchrotron radiation. The energy of the electrons after they transit 
through the last undulator is fully recovered, and the electrons are recirculated. In Fig. 2, a general scheme for such a device is shown [15]. The concept has been tested in the infrared wavelength range at Jefferson Laboratory [16]. Scientists and engineers at Berkeley [17], Brookhaven [18], and Cornell [19] have developed new proposals for ERL-based synchrotron radiation sources in the X-ray range. These new sources permit one to realize higher average brilliance (and hence higher coherent flux) at x-ray wavelengths than are possible with third-generation synchrotron sources, and they deliver x-ray pulses that could be as short as few hundred fs.

An x-ray FEL can be designed to provide full transverse coherence, higher average brilliance, and pulses that are even shorter than 100 fs. These unique radiation characteristics are achieved by accelerating high-energy, low-emittance, high-peak-current compressed electron bunches through a long undulator. As the electron bunch travels through the undulator, it interacts with the radiation, acquiring a longitudinal density modulation at x-ray wavelengths. These modulated microbunches emit x-rays coherently and collectively enhancing the intensity by 6-7 orders of magnitude compared to conventional spontaneous radiation from the undulator (see Fig. 3). This enhancement process is called self-amplified spontaneous emission (SASE) [20-22]. A seeding scheme, which is being developed, will provide longitudinal coherence. The SASE FEL concept has not yet been demonstrated at x-ray wavelengths. Recently, work at Argonne[23] and Hamburg[24] has shown saturation in SASE process in the $100 \mathrm{~nm}$ range. These studies have lent considerable confidence to the understanding of SASE process and led to new proposals to build vacuum ultraviolet (VUV) and x-ray FEL sources. These include the VUV sources BESSY SASE-FEL in Berlin [25] and TTF-2 SASE-FEL in Hamburg [26]. In the x-ray range, the proposals include the LCLS at Stanford [26,27], TESLA FEL at Hamburg [26,28], and SPring-8 Compact SASE FEL in Japan [29]. 


\section{Characteristics of Future Sources}

In understanding the performance of the future sources, it is essential to define the characterizing parameters. The average brilliance (B) is the photon flux (F) per unit transverse phase space volume given by $\mathrm{B}=\mathrm{F} /\left[(2 \pi)^{2} \varepsilon_{\mathrm{x}} \varepsilon_{\mathrm{y}}\right.$, where $\varepsilon_{\mathrm{x}}$ and $\varepsilon_{\mathrm{y}}$ are the horizontal and vertical beam emittances. The peak brilliance (BP) gives us the photon flux per pulse in the 6-D phase space volume, which includes both transverse and longitudinal directions. Assuming Gaussian optics, $\mathrm{BP}=\left[(2.35)^{2} /(2 \pi)\right] \mathrm{B} /(\tau \mathrm{f})$, where $\tau$ and $f$ are the electron pulse length and pulse frequency in a linac or a storage-ring. The value of the coherent flux (FC) is obtained from the average brilliance, $\mathrm{FC}=\mathrm{B}(\lambda / 2)^{2} \times 10^{-8}$, where $\lambda$ is the wavelength of x-rays. In Table 1, a comparison of critical parameters in the x-ray range for the existing third-generation synchrotron facilities, as well as ERLs and FELs, is given.

\section{Future Impact of New Sources on Materials Research}

As we look ahead at these new sources, it is clear from Table 1 that there is a major revolution in source characteristics compared to currently used synchrotron radiation sources, which will have a major impact on the future scientific research in the areas of biological science, chemical science, astrophysics, warm-dense matter physics, condensed matter and materials sciences [25, 28, 30, 31]. In materials and condensed matter, the broad research focus will be on experiments that have been marginal at thirdgeneration sources and will capitalize on the 2 to 4 orders-of-magnitude increase in average brilliance. However a new class of experiments will benefit from higher peak brilliance, higher coherent flux, and fs pulse length. The pump-probe studies will be able to address both equilibrium and non-equilibrium processes. Most of these experiments have the promise to revolutionize our understanding of fundamental correlations in materials. While many of these advances have been discussed elsewhere $[25,27,28]$, here a few examples of physical processes in materials in the fs time domain are presented. Full understanding of these will require next-generation sources with higher peak brilliance and coherence. 
Nonthermal Melting of Solids:

What is the nature of melting of solids when the duration of melting pulse is as short as the vibrational period of atoms $(\approx 100 \mathrm{fs})$ ? This question has been addressed for nearly a decade using a variety of measurement techniques, but a full understanding of the operative mechanisms requires structure information in the femtosecond time domain. To shed some light, recently, plasma-based subpicosecond $\mathrm{X}$-ray pulses have been used in pump-probe studies of semiconductors excited by deposition of $30 \mathrm{fs}$ laser pulses of different fluence $[32,33]$. Though weak in intensity, the x-ray probes have provided some insight into the melting phenomena following the excitation. As opposed to thermal melting, short optical laser pulses rapidly excite a large population of electrons initially bonded to atoms into the conduction band of the materials, producing electron-hole plasma. This immediately produces repulsive interatomic forces, destroying the cohesion of the lattice structure. The velocity acquired by the atoms under the action of these forces destroys the crystalline order within a few fs and produces a liquid layer. The observed disorder in the lattice is not attributable to slower thermal processes that convert electronic energy into phonons. Hence this new process is called non-thermal melting. As time progresses, various mechanisms of melting and recrystallization become operative determined by electron-phonon relaxation processes. The nonthermal melting phenomenon and various relaxation mechanisms are likely to be investigated in detail in condensed matter, chemical, and biological materials when the ERL and FEL sources become available. There are great opportunities also to compare the measurements of these processes to computational simulations [34].

\section{Femtosecond Structural Dynamics in Highly Correlated Materials:}

Vanadium dioxide $\left(\mathrm{VO}_{2}\right)$ is a prototypical highly correlated system in which the structural phase change accompanies a metal-insulator transition due to changes in electronic band structure [35]. This phase transition has always been thought to be thermally initiated. Recent work focusing on initiating this transition has been in the fs time domain [36]. The experiment involved changing the band gap in $\mathrm{VO}_{2}$ by pumping the system with a 50 fs laser pulse initiating the interband electronic excitation. This was 
followed by time-delayed x-ray diffraction measurements using a plasma source producing a few hundred femtosecond, 8-keV pulses. Remarkably, the structural phase transition occurred even over time periods well before the photoexcitation processes were thermalized. This was the first demonstration of an unusual microscopic process involving transition between two equilibrium states separated by a nonequilibrium state. Detailed understanding of this process in the future will require new sources with higher photon flux per bunch.

Electron-Mediated Catalysis Initiated by Femtosecond Infrared Pulse:

In a traditional picture, the chemical reactions in a catalytic process take place when the system is excited thermally via phonons that drive the system across the activation barrier in the electronic ground state. A perfect example is desorption of only $\mathrm{CO}$ from a Ru (0001) metal surface coadsorbed with $\mathrm{CO}$ and $\mathrm{O}_{2}$ molecules when heated [37]. On the other hand, when the same system is excited with femtosecond infrared laser pulse, the barrier for $\mathrm{O}$ activation is overcome by coupling to hot electrons produced by the fs laser pulse, so that $\mathrm{CO}_{2}$ is formed and desorbed [38] - the conclusion being that the oxidation reaction is electron mediated. Hot electrons from $\mathrm{Ru}$ produced by the $800 \mathrm{~nm}$ fs pulse populate the antibonding orbitals of Ru-O bond, and this, in turn, increases the Ru-O bond length. An experimental measurement of $\mathrm{Ru}-\mathrm{O}$ distance as a function of time following the laser excitation in the subpicosecond domain would be crucial in defining the role of hot electrons in the chemical activity of the absorbate. The femtosecond x-ray pulses from a future ERL or FEL would be able to provide a definitive picture of these catalytic processes.

Imaging of Single Molecules and Nanoparticles:

Structures of complex materials including proteins are being investigated at third-generation synchrotron radiation sources at near-atomic or atomic resolution using both diffraction and imaging, and these advances will have a major impact on nanoscience in the future. The method demands high-quality crystals of sufficient size. However, as interest has been growing in modern technological systems 
involving a single molecule and small clusters, it is important to address the applicability of known methodologies. Modern day x-ray optics will allow focusing of the beam down to $30-50 \mathrm{~nm}$, and this will produce enormous radiation damage, especially with future sources, such as x-ray FELs. Fortunately, theoretical simulations by Hajdu and collaborators show that, within about 10 - 100 femtoseconds, biomolecules and small particles can withstand an x-ray intensity of $\sim 3.8 \times 10^{6}$ photons $/ \AA^{2}$ with minimal structural changes [39]. A 2D diffraction pattern can hence be obtained from a single ultrashort exposure before the radiation damage manifests itself. From a set of such diffraction patterns covering 3D reciprocal space by rotating the sample, the structure of the molecule can be obtained if phase information is available. The phase problem can be solved by using the oversampling method, which was first demonstrated by successfully converting an experimental image from a noncrystalline specimen to the structure of the specimen [40]. In a simulation of x-ray scattering from a particle made up of about 100 rubisco molecules using a 10-fs 1.5 - $\AA$ x-ray FEL pulse with 2 x $10^{12}$ photons, and analyzing the data using the oversampling method, the 3D electron density map was reconstructed from the noisy 3D diffraction patterns [41]. The applicability of the femtosecond 2D diffraction, coupled to oversampling methodology has a lot of promise for the study of nanoclusters of materials even when the sample is noncrystalline and contains imperfections.

\section{Conclusions}

In this paper a review of the potential impact of future synchrotron radiation sources on materials science is briefly summarized. Highly coherent and high-intensity ultrashort x-ray pulses from ERLs and x-ray FELs will be the key in the study of both equilibrium and nonequilibrium physics in materials. The availability of x-ray FELs will lead to the discovery and measurement of many physical correlations that manifest below 100 femtoseconds through pump-probe experiments.

\section{ACKNOWLEDGMENTS}

This work is supported by U.S. DOE-BES under contract No. W-31-109-ENG-38. 
Figure Captions:

Fig. 1 Conjugate space ( $\omega, t$; Q,r) showing the resolution of various tools probing materials using $\mathrm{x}$ rays. The domain open to next-generation sources is indicated.

Fig. 2 Conceptual layout of an energy recovery linac (ERL) based x-ray source [19].

Fig. 3 Conceptual layout of a SASE-based x-ray FEL. 
Table 1. Comparison of average values of some of the parameters for the third-generation synchrotron radiation sources, proposed ERL sources and proposed FELs in the x-ray wavelength range.

\begin{tabular}{|c|c|c|c|}
\hline Parameters & $\begin{array}{c}\text { Third- } \\
\text { Generation } \\
\text { Sources } \\
\end{array}$ & ERLs & FELS \\
\hline Electron Energy $(\mathrm{GeV})$ & $5-8$ & $5-7$ & $15-25$ \\
\hline Avg. Current (mA) & $100-300$ & $10-100$ & $10^{-4}-10^{-1}$ \\
\hline Charge per bunch (nC) & $0.3-14$ & $0.01-0.1$ & 1.0 \\
\hline$\varepsilon_{\mathrm{x}}(\mathrm{nm}-\mathrm{rad})$ & $3-8$ & $0.01-0.15$ & $0.02-0.05$ \\
\hline$\varepsilon_{\mathrm{y}}(\mathrm{nm}-\mathrm{rad})$ & $0.003-0.08$ & $0.01-0.15$ & $0.02-0.05$ \\
\hline Beam Shape $\left(\varepsilon_{\mathrm{x}} / \varepsilon_{\mathrm{y}}\right)$ & Flat $(\approx 100)$ & Round $(\approx 1)$ & Round $(\approx 1)$ \\
\hline $\begin{array}{l}\text { Bunch Length, } \tau \\
\text { (fwhm-fs) }\end{array}$ & $\begin{array}{c}30,000- \\
70,000 \\
\end{array}$ & $<300$ & $50-200$ \\
\hline $\begin{array}{l}\text { Bunch Frequency, f } \\
(\mathrm{Hz})\end{array}$ & $2 \times 10^{6}-3.4 \times 10^{8}$ & $1.3 \times 10^{9}$ & $120-5.6 \times 10^{4}$ \\
\hline Undulator Period $(\mathrm{cm})$ & $1.8-5.4$ & 1.7 & $3-5$ \\
\hline Undulator Length (m) & $2.4-25$ & 25 & $30-100$ \\
\hline First Harmonic (keV) & $2.5-15$ & $6-15$ & $8-12$ \\
\hline $\begin{array}{l}\text { Ave. Flux, F } \\
(\mathrm{ph} / \mathrm{s} / 0.1 \% \mathrm{BW})\end{array}$ & $10^{15}-10^{10}$ & $10^{15}-10^{10}$ & $10^{14}-10^{17}$ \\
\hline $\begin{array}{l}\text { Ave. Brilliance, B } \\
\left(\mathrm{ph} / \mathrm{s} / 0.1 \% \mathrm{BW} / \mathrm{mm}^{2} / \mathrm{mr}\right. \\
\left.\mathrm{ad}^{2}\right)\end{array}$ & $10^{19}-10^{21}$ & $10^{22}-10^{23}$ & $10^{22}-10^{25}$ \\
\hline $\begin{array}{l}\text { Coherent Flux, FC } \\
(\mathrm{ph} / \mathrm{s} / 0.1 \% \mathrm{BW})\end{array}$ & $10^{11}-10^{15}$ & $10^{13}-10^{14}$ & $10^{14}-10^{17}$ \\
\hline $\begin{array}{l}\text { Peak Brilliance, BP } \\
\left(\mathrm{ph} / \mathrm{s} / 0.1 \% \mathrm{BW} / \mathrm{mm}^{2} / \mathrm{mr}\right. \\
\left.\mathrm{ad}^{2}\right)\end{array}$ & $10^{22}-10^{23}$ & $10^{25}-10^{26}$ & $10^{33}-10^{34}$ \\
\hline $\begin{array}{l}\text { Peak Flux } \\
(\mathrm{p} / \mathrm{s} / 0 / 1 \% \mathrm{BW}) \\
\end{array}$ & $10^{17}-10^{18}$ & $10^{18}-10^{19}$ & $10^{24}-10^{25}$ \\
\hline $\begin{array}{l}\text { Photons/bunch/0.1\%B } \\
\text { W }\end{array}$ & $10^{7}-10^{8}$ & $10^{6}-10^{7}$ & $10^{12}-10^{13}$ \\
\hline Peak E-field $(\mathrm{V} / \mathrm{cm})$ & $10^{5}-10^{6}$ & $10^{6}-10^{8}$ & $10^{10}-10^{11}$ \\
\hline
\end{tabular}


References:

1. L. Van Hove, Phys. Rev. 95 (1954) 249.

2. W. Schulke, Inelastic Scattering by Electronic Excitations in Handbook of Synchrotron Radiation, vol 3, ed G. Brown and D. E. Moncton, Elsevier Publ., Amsterdam, 1991; E. Burkel, Rep. Prog. Phys. 63 (2000) 171.

3. S. K. Sinha, J. Phys: Condens. Matter 13 (2001) 7511.

4. E. E. Alp, W. Sturhahn, H. Sinn, T. Toellner, M. Hu, J. Sutter, A. Alatas, , Proc. Internat. Conf. X-ray and Inner Shell Processes, R. W. Dunford, et al., eds., 506, AIP, (2000) pp. 479-485.

5. W. Sturhahn, E. E. Alp, T. S. Toellner, P. Hession, M. Hu, J. Sutter, Hyperfine Interact. 113 (1998) 47.

6. L. B. Lurio, D. Lumma, M. A. Borthwick, P. Falus, S. G.J. Mochrie, J. F. Pelletier, M. Sutton, Synchrotron Radiat. News 13(2) (2000) 28.

7. S. Techert, F.Schotte, and M. Wulff, Phys. Rev. Lett. 86 (2001) 2030.

8. D. Lumma, M. A. Borthwick, P. Falus, L. B. Lurio, S. G. J. Mochrie, Phys. Rev. Lett. 86 (2001) 2042.

9. J. R. Helliwell and P. M. Rentzepis Time-Resolved Diffraction, Clarendon-Press, Oxford, 1997.

10. A. Rundquist, C, G. Dufree, III, C. Zenghu, C. Herne, S. Bakus, M. M. Murnane, and H. C. Kapteyn, Science 289 (1998) 1412.

11. Ch. Rose-Petruck, T. Guo, R. Jimenez, F. Raksi, J. Squier, B. Walker, K. R. Wilson, and C. P. J. Barty, Nature 398 (1999) 310.

12. H. Winick, J. Synchrotron Rad., 5 (1998), 168.

13. A. Ropert, J. M. Filhol, P. Elleaume, L. Farvacque, L. Hardy, J. Jacob, U. Weinrich, Proc. of EPAC 2000, Vienna, Austria, p. 83.

14. M. Tigner, Nuovo Cimento, 37 (1965) 1228. 
15. S. M. Gruner and M. Tigner, Eds. CHESS Technical Report Memo 01-003, 2001, Cornell High Energy Synchrotron Source, Cornell University, Ithaca, NY.

16. G. R. Neil, C. L. Bohm, S. V. Bensen, G. Biallas, D. Douglas, H. F. Dylla, R. Evans, J. Fugitt, A. Grippo, R. Hill, K. Jordan, R. Li, L. Merminga, P. Piot, J. Preble, M. Shinn, T. Soggins, R. Walker, B. Yunn, Phys. Rev. Lett. 84 (2000) 662.

17. H. A. Padmore, R. W. Schoenlein, and A. A. Zholents, Synchrotron Rad. News 14(2) (2001) 26.

18. I. Ben-Zvi, and S. Krinsky, Synchrotron Rad. News 14(2) (2001) 20.

19. D. Biderbach, I. Bazarov, K. Finkelstein, S. Gruner, G. Krafft, L. Merminga, H. Padamsee, Q. Shen, C. Sinclair, M. Tigner, and R. Talman, Synchrotron Rad. News 14(3) (2001) 12.

20. A. M. Kondratenko and E. L. Saldin, Part. Accelerators 10 (1980) 207.

21. Ya. S. Derbenev, A. M. Kondratenko, and E. L. Saldin, Nucl. Instrum. Methods A193 (1982) 415.

22. R. Bonifacio, C. Pellegrini, and L. Narducci, Opt. Commun. 50 (1984) 373.

23. S. V. Milton, et al., Phys. Rev. Lett. 85 (2000) 988; S. V. Milton et al., Science 292 (2001) 2037.

24. J. Andruszkow, et al., Phys. Rev. Lett. 85 (2000) 3825.

25. W. Eberhardt, this conference.

26. H.-D. Nuhn and J. Rossbach, Synchrotron Rad. News 13(1) (2000) 18.

27. The LCLS Design Study Group, Linac Coherent Lighy Source (LCLS) Design Study Report, SLAC-R-521, UC-414 (1998).

28. R. Brinkmann, G. Matterlik, J. Rossbach and A. Wagner, Eds. DESY 1997-048 and ECFA 1997182 (1997).

29. T. Shintake, H. Matsumoto, T. Ishikawa, and H. Kitamura, Proc. $46^{\text {th }}$ SPIE Meeting, 2001, San Diego (in press)

30. G. Williams, this conference 
31. G. Shenoy and J. Stohr, Eds., LCLS - The First Experiments, SLAC Publication, 2000; G. Materlik and Th. Tschentscher, Eds., TESLA - The X-ray Free Electron Laser, DESY 2001-011, TESLA-FEL 2001-05, 2001.

32. C. W. Siders, A. Cavalleri, K. Sokolowski-Tinten, Cs. Toth, T. Guo, M. Kammler, M. Horn von Hoegen, K. R. Wilson, D. von der Linde, and C. P. J. Barty, Science 286 (1999) 1340.

33. A. Rousse, C. Rischel, S. Fourmaux, I. Uschmann, S. Sebban, G. Grillon, Ph. Balcou, E. Forster, J. P. Gendre, P. Aubebert, J. C. Gauthler, and D. Hulln, Nature 410 (2001) 65.

34. P. L. Silvestrelli, A. Alavi, M. Perrinello, and D. Frenkel, Phys. Rev. B 56 (1997) 3806; P. Stampfli and K. H. Bennemann, Phys. Rev. B 49 (1994) 7299.

35. M. Imada, A. Fujimori, and Y. Tokura, Rev. Mod. Phys. 70 (1998) 1034.

36. A. Cavalleri, Cs. Toth, C. W. Siders, J. A. Squier, F. Raksi, P. Forget, and J. C. Kieffer, Phys. Rev. Lett. 87 (2001) 237401.

37. A. Bottcher, H. Niehus, S. Schwegman, H. Over, and G. Ertl, J. Phys. Chem. B 101 (1997) 11185.

38. M. Bonn, S. Funk, Ch. Hess, D. N. Denzler, C. Stampfl, M. Scheffler, M. Wolf, and G. Ertl, Science, 285 (1999) 1042.

39. R. Neutze, R. Wouts, D. Spoel, E. Weckert, E. and J. Hajdu, Nature 406 (2000) 752.

40. J. Miao, P. Charalambous, J. Kirz, and D. Sayre, Nature 400 (1999) 342.

41. J. Miao, K. O. Hodgson, and D. Sayre, Proc. Natl. Acad. Sci. 98 (2001) 6641 


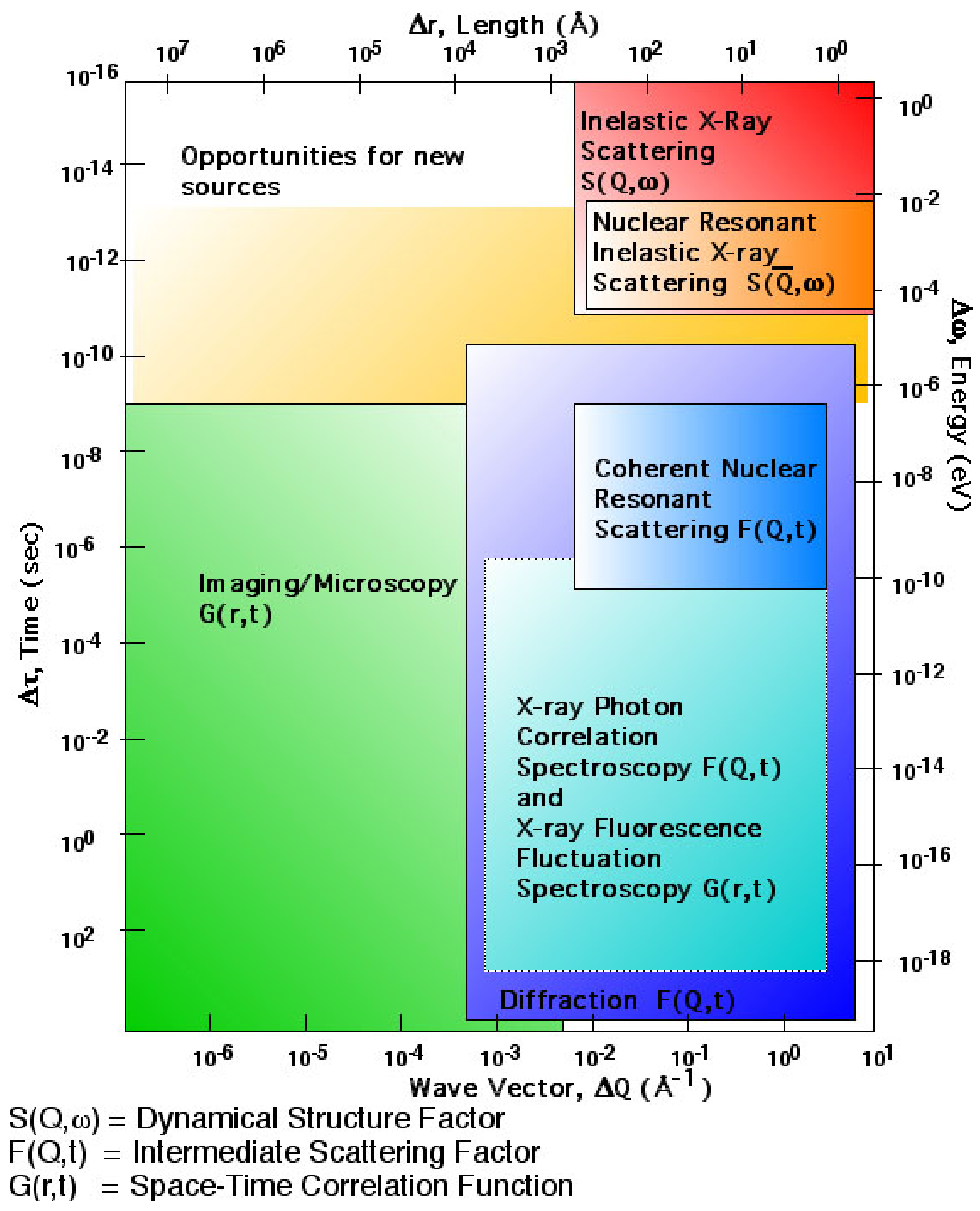


Figure 2. Shenoy:

\section{Electron Recovery Linac (ERL) Concept (Cornell)}

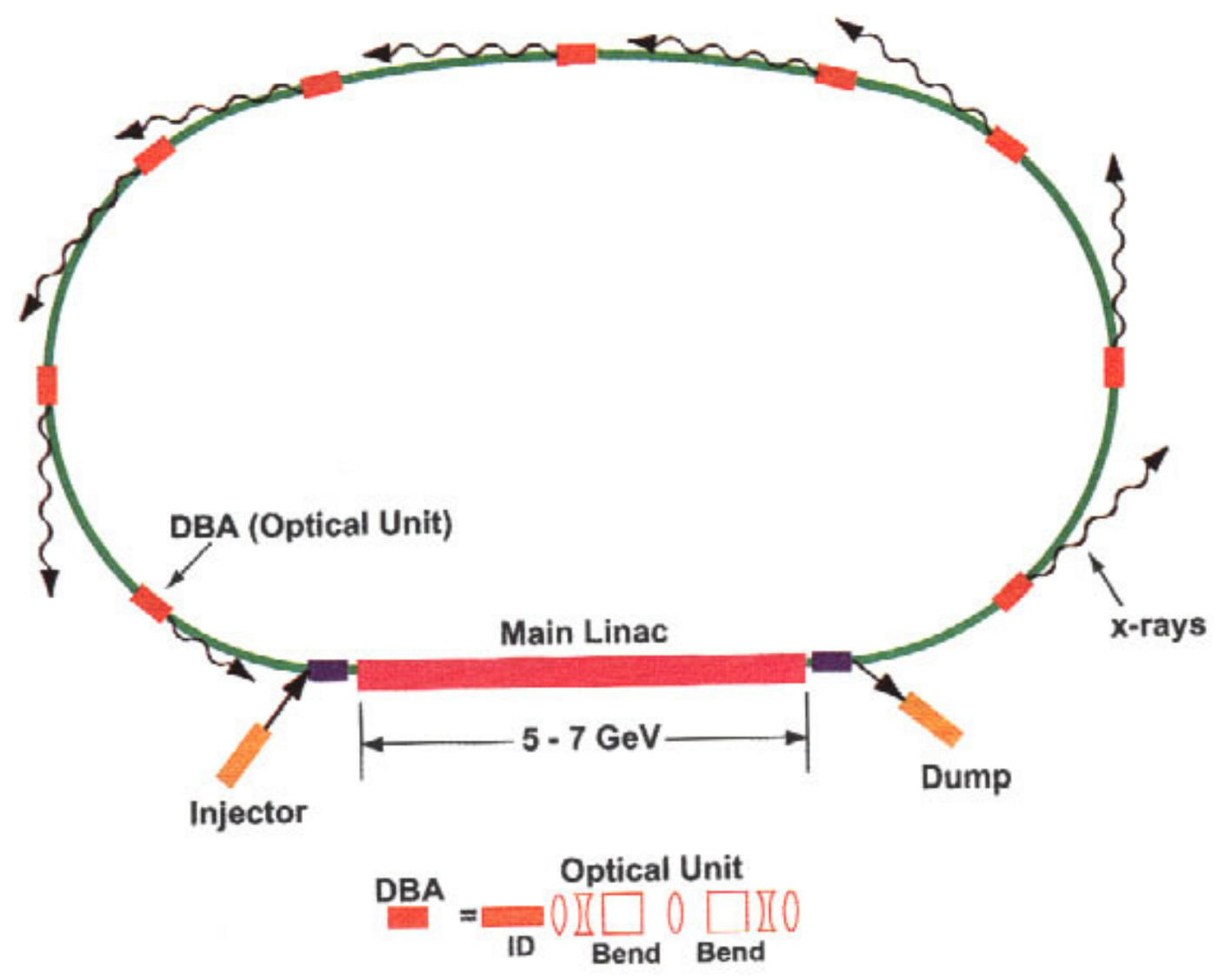


Figure 3 Shenoy:

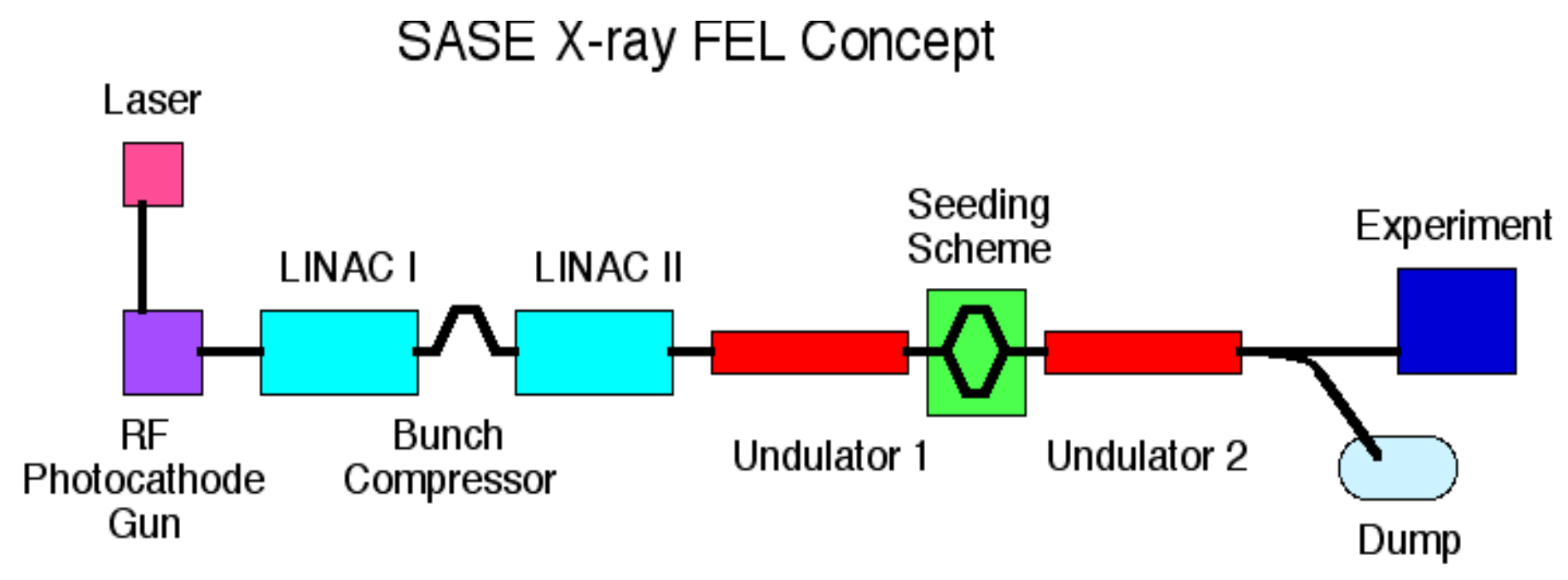

